$R M x A C, \mathbf{5 3}, 67-74(2021)$

(c) 2021: Instituto de Astronomía, Universidad Nacional Autónoma de México

https://doi.org/10.22201/ia.14052059p.2021.53.17

\title{
GALAXY-TARGETED ROBOTIC TELESCOPE FOLLOW-UP OF GRAVITATIONAL WAVE EVENTS
}

\author{
L. Salmon ${ }^{1}$, L. Hanlon ${ }^{1}$, R. M. Jeffrey ${ }^{1}$, and A. Martin-Carrillo ${ }^{1}$
}

\section{RESUMEN}

Las redes de telescopios robóticos están bien equipadas para responder rápidamente a eventos transitorios. Sin embargo, en la nueva era de astronomía de multi-mensajeros, la búsqueda de las contrapartidas electromagnéticas de ondas gravitacionales supone un nuevo reto. En particular, las fuentes pueden estar a grandes distancias; ser débiles; tener una localización pobre, abarcando una gran parte del cielo; y su brillo puede decaer muy rápidamente. Así pues, la búsqueda eficiente de estas contrapartidas require cubrir una gran parte del cielo y/o el apuntado efectivo a las galaxias que puedan albergar la fuente. Este artículo presenta un algoritmo para clasificar las galaxias huéspedes candidatas y su implementación en una interfaz web pública que responde de forma automática después de cada detección de ondas gravitacionales. La página web está disponible al público en https://gwtool.watchertelescope.ie/.

\section{ABSTRACT}

Robotic telescopes and networks are well equipped to respond rapidly to transient events. However, the era of multi-messenger astronomy presents new challenges in the search for electromagnetic counterparts to gravitational wave events. Specifically, these sources can be distant, faint, poorly localised, and quickly decaying. Efficiently searching for counterparts requires coverage of large localisation regions and/or targeted observations. This paper presents a galaxy retrieval and ranking algorithm for targeted observations, and a public web interface to retrieve ranked galaxy lists following a gravitational wave event. The website is publicly available at https://gwtool.watchertelescope.ie/.

Key Words: gamma-ray burst: general — gravitational waves — methods: miscellaneous

\section{INTRODUCTION}

The era of multi-messenger astronomy presents significant challenges to current telescope follow-up strategies. Electromagnetic counterparts are expected from the Neutron Star - Neutron Star (NSNS) and Neutron Star - Black Hole (NS-BH) merger events detected by the current gravitational wave (GW) observatories - LIGO (Aasi et al. 2015) and Virgo (Acernese et al. 2015). During the LIGO Observing Run 3 (O3), which began in April 2019 and lasted 1 year, merger events were detected more frequently and at greater distances than previously. There is an expected rate of 1-50 NS-NS mergers per year out to $170 \mathrm{Mpc}$ during O3. The rate of NS-BH events is uncertain but likely to be lower (Abbott et al. 2018).

The localisation regions of GW events, calculated via triangulation by the growing network of GW detectors, can vary greatly in size. The challenge is to search, identify and monitor these fast-decaying and poorly localised sources with the current telescope infrastructure. An efficient follow-up strategy must

\footnotetext{
${ }^{1}$ School of Physics, University College Dublin, Belfield, Dublin 4, Ireland (lana.salmon@ucdconnect.ie).
}

be put in place by existing telescopes, by considering the outputs and limitations of GW detectors, and the expected counterpart properties of these events.

The classification, localisation region and posterior mean distance of a gravitational wave event drive the decision to follow-up an event, and the strategy for doing so. In August of 2017, a nearby $(40 \mathrm{Mpc})$ NS-NS merger was detected by Advanced LIGO (Aasi et al. 2015). The event was very well localised with a final $90 \%$ localisation region of 28 $\operatorname{deg}^{2}$ (Abbott et al. 2017). The detection of an almost coincident burst of gamma-rays by FermiGBM and Integral SPI-ACS $\sim 1.7$ seconds after the event helped to constrain its location (Goldstein et al. 2017; Savchenko et al. 2017). An international follow-up campaign by ground and space-based observatories led to the detection of panchromatic afterglow and kilonova emission at $\sim 2 \mathrm{kpc}$ from the galaxy NGC4993 (Abbott et al. 2017; Andreoni et al. 2017; Arcavi 2017; Coulter et al. 2017; Cowperthwaite et al. 2017; Drout et al. 2017; Evans et al. 2017; Kilpatrick et al. 2017; McCully et al. 2017; Pian et al. 2017; Smartt et al. 2017; Tanvir et al. 2017; Valenti et al. 2017). 
NS-NS and NS-BH mergers are thought to be the progenitors of short Gamma-Ray Bursts (GRBs; Belczynski et al. 2006; Eichler et al. 1989; Narayan et al. 1992), and many short GRBs have been detected in galaxies lacking of star formation (Fong et al. 2016). GRBs release energy in the form of a collimated jet, which interacts with the surrounding medium to create a panchromatic afterglow (Berger 2014). The radioactive decay of neutron rich ejecta in the material surrounding the NS-NS/NSBH merger produces NIR/optical emission, known as a kilonova (Metzger 2017).

The detected counterpart properties vary greatly depending on the observer's viewing angle and properties of the ejecta. An off-axis viewing angle can suppress the detection of the GRB afterglow, especially at early times when the jet opening angle is small, allowing the isotropic kilonova emission to be detected (Granot et al. 2002; Hotokezaka \& Piran 2015; Jin et al. 2018; Kathirgamaraju et al. 2018; Metzger \& Berger 2012). In the case of GW170817, one favoured model involves a strucured jet viewed off-axis, allowing the kilonova to be observed at early times (Alexander et al. 2018; Fong et al. 2019; Lazzati et al. 2018; Margutti et al. 2017; Mooley et al. 2018; Troja et al. 2018; Wu \& MacFadyen 2018). The composition of the ejecta affects the evolution of kilonova light curves. Lanthanide rich (i.e. neutron heavy) ejecta creates a red kilonova, peaking over days to a week in the NIR bands. A blue kilonova is expected from lighter, low opacity ejecta with a peak in optical bands over 1 day (Barnes \& Kasen 2013; Kasen et al. 2013; Li \& Paczyński 1998; Metzger 2017; Roberts et al. 2011; Tanaka \& Hotokezaka 2013).

GW170817 demonstrated the fast decay of the counterpart - the kilonova associated with GW170817 decayed at a rate of $1.1 \mathrm{mag} /$ day in the $\mathrm{r}$ band (Valenti et al. 2017). The location of this emission must be pinpointed as early as possible, before it has faded below detectability. Early detection, however, is hindered by the large localisation regions (typically $\sim 20-1000 \mathrm{deg}^{2}$; Abbott et al. 2018) provided by the current detector network. The initial 99\% localisation region for S190510g can be seen in Figure 1, demonstrating a particularly complex localisation region made up of multiple, oddly-shaped regions which must be observed in a particular order, defined by the observing strategy used.

This paper presents a galaxy retrieval algorithm and public web interface to assist telescopes in conducting galaxy-targeted follow-up of gravitational wave events.

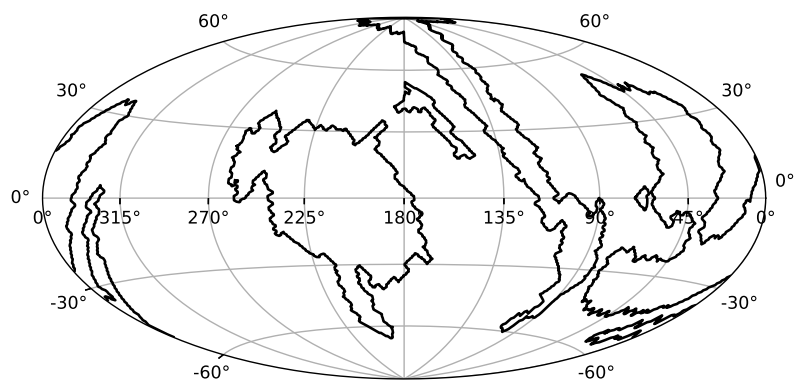

Fig. 1. Contours enclosing $99 \%$ of the localisation region for S190510g. The localisation region for this event is large, complex and disjointed, presenting a challenge to GW follow-up programmes attempting to detect an electromagnetic counterpart.

\section{FOLLOW-UP STRATEGIES FOR ROBOTIC TELESCOPES AND NETWORKS}

Many robotic telescopes and networks conduct follow-up campaigns to transient events such as GRBs. The telescopes are triggered by the NASA Gamma-Ray Coordinates Network $(\mathrm{GCN})^{1}$ system. The first detection of a GRB afterglow in 1997 made the case for robotic telescopes to survey GRB error boxes as soon as possible after an event (Costa et al. 1997; Frail et al. 1997; Van Paradijs et al. 1997). GRB localisations (Connaughton et al. 2015; Goad et al. 2007; Mereghetti 2013) and telescope response times have improved to meet this challenge, with seconds response time in some cases (Pozanenko et al. 2003). The community must make use of this optimised infrastructure and adapt it to meet the new challenges related to the follow-up of poorly localised gravitational wave events, whose alerts are also distributed using the GCN alert system.

Table 1 shows the fields of view of a sample of robotic telescopes and networks. The number of tiles required to cover the entire $99 \%$ localisation region for the initial sky map for the likely NS-BH event S190814bv, calculated using the sky_tiling ${ }^{2}$ code, is shown in column 3 . The total number of tiles is compared to the number of tiles required to cover all galaxies within the $99 \%$ localisation region (column 4). Wide field telescopes make use of their large field of view to conduct tiled observations of GW localisation regions, whereby the whole region is covered by tiles equal to the field of view of the telescope. The efficient placement and order of observation of the tiles can shorten the time required to cover the whole region and identify the counterpart. For a review of these tiling optimisation strategies, see Coughlin et al. (2018); Rana et al. (2017).

\footnotetext{
${ }^{1}$ https://gcn.gsfc.nasa.gov/lvc.html

${ }^{2}$ https://github.com/shaonghosh/sky_tiling.git
} 
TABLE 1

COMPARISON OF WIDE AND NARROW FIELD ROBOTIC TELESCOPES

\begin{tabular}{|c|c|c|c|}
\hline Telescope & $\begin{array}{l}\text { FoV per } \\
\text { telescope } \\
\left(\operatorname{deg}^{2}\right)\end{array}$ & $\begin{array}{l}\# \quad \text { tiles } \\
\text { to cover } \\
99 \% \\
\text { S190814bv }\end{array}$ & $\begin{array}{l}\# \quad \text { tiles } \\
\text { to cover } \\
\text { galaxies } \\
\text { in } \quad 99 \% \\
\text { S190814bv }\end{array}$ \\
\hline ASSASN-1 & 4.5 & 494 & 494 \\
\hline ATLAS & 29 & 98 & 98 \\
\hline $\begin{array}{l}\text { MASTER } \\
\text { WFC }\end{array}$ & 24 & 103 & 103 \\
\hline $\begin{array}{l}\text { PanSTARRS- } \\
1\end{array}$ & 7 & 149 & 149 \\
\hline BOOTES & 0.16 & 12,803 & 9248 \\
\hline Watcher & 0.16 & 12,803 & 9248 \\
\hline
\end{tabular}

Telescopes with small fields of view cannot cover these regions in sufficiently short times. A targeted approach, which involves only observing galaxies in the localisation region, can reduce the number of pointings required by a factor of 10-100 (Gehrels et al. 2016). To optimise this strategy even further, the known properties of these galaxies and the LIGO/Virgo probabilities can be combined to perform a ranking of galaxies. There are galaxy ranking algorithms currently in use by telescopes and networks, which perform rankings of fields of view or individual galaxies based on LIGO/Virgo probabilities and the luminosities and/or distances of galaxiessee Dobie et al. (2019); Evans et al. (2016); Kasliwal et al. (2017); Klingler et al. (2019); Rana \& Mooley (2019); Yang et al. (2019). This work makes use of the ranking algorithm presented by Arcavi et al. (2017), which ranks individual galaxies based on LIGO/Virgo probabilities and the distance and luminosity of the galaxies. This approach is more suitable to a strategy for narrow-field telescopes which do not require ranking of large fields containing many galaxies.

\section{GALAXY RETRIEVAL ALGORITHM}

Version 1 (V1) of the galaxy retrieval algorithm is detailed in this section and a brief discussion of Version 0 (V0) of the algorithm is presented in $\S 5.1$. Both versions of the algorithm can be found on separate branches on GitHub ${ }^{3}$. The galaxy retrieval algorithm is developed in Python 3 to take as input the GW sky map and galaxy catalogue, and output a list of ranked galaxies within the localisation region.

\footnotetext{
${ }^{3}$ https://github.com/Lanasalmon/HOGWARTs
}

\subsection{Inputs}

\subsubsection{Sky map}

The decision to launch a follow-up campaign relies on the information contained in the GW trigger; namely, the event classification (e.g. NS-NS), False Alarm Rate (FAR), and sky map FITS file. The 'sky map' is a FITS file which represents a map of the whole sky, comprising pixels generated by the BAYESTAR (Singer \& Price 2016) or the LALInference algorithm (LIGO Scientific Collaboration 2018; Veitch et al. 2015) and stored in a HEALPix projection (Górski et al. 2005). The resolution of sky maps can vary, and each sky map contains hundreds of thousands of pixels. Each pixel contains 4 parameters:

- $p_{\text {loc }}$ - The probability that the source is in the pixel.

- $\mu_{\text {dist }}$ - Distance estimate.

- $\sigma_{\text {dist }}$ - Standard deviation on the distance estimate.

- $N_{\text {dist }}$ - Normalisation constant.

Additionally, the sky map header contains the posterior mean distance (DISTEST) and standard deviation (DISTSTD) marginalised over the whole sky.

\subsubsection{Galaxy catalogue}

Galaxy catalogues such as the Gravitational Wave Galaxy Catalogue (GWGC; White et al. 2011) and the Galaxy List for the Advanced Detector Era ${ }^{4}$ (GLADE; Dálya 2018) have been developed for use in gravitational wave follow-up. GLADE contains galaxies from 2MASS (Skrutskie et al. 2006), HyperLEDA (Makarov et al. 2014) and GWGC. A comparison of the GWGC, GLADE V1 and GLADE V2 catalogues can be seen in Table 2. These catalogues suffer from incompleteness at distances beyond $\sim 100 \mathrm{Mpc}$. A measure of completeness of these catalogues is plotted in Figure 2. The completeness is calculated by comparing the cumulative B luminosity within different distances in the catalogue to the B luminosity expected from a homogeneous complete galaxy catalogue given by Kopparapu et al. (2008).

We use GLADE V2 in our galaxy retrieval algorithm as:

- GLADE V1 and V2 contain more galaxies and are more complete than GWGC.

\footnotetext{
${ }^{4}$ http://glade.elte.hu/index.html
} 
TABLE 2

COMPARISON OF THE PROPERTIES OF GWGC, GLADE V1 AND GLADE V2

\begin{tabular}{lcccc}
\hline Property & GWGC $^{\mathrm{a}}$ & GLADE V1 $^{\mathrm{a}}$ & GLADE V1 $^{\mathrm{ab}}$ & GLADE V2 $^{\mathrm{a}}$ \\
\hline \# galaxies & 53,312 & $1,918,147$ & $1,490,234$ & $1,613,030$ \\
Parameter estimation & No & Yes & No & No \\
$100 \%$ completeness distance $(\mathrm{Mpc})$ & 31 & 73 & 37 & 37 \\
$50 \%$ completeness distance $(\mathrm{Mpc})$ & 102 & 328 & 164 & 148 \\
\hline
\end{tabular}

${ }^{\mathrm{a}}$ Including only galaxies with $\mathrm{B}$ magnitude and distance values.

${ }^{\mathrm{b}}$ Including only galaxies whose parameters have not been estimated via regression.

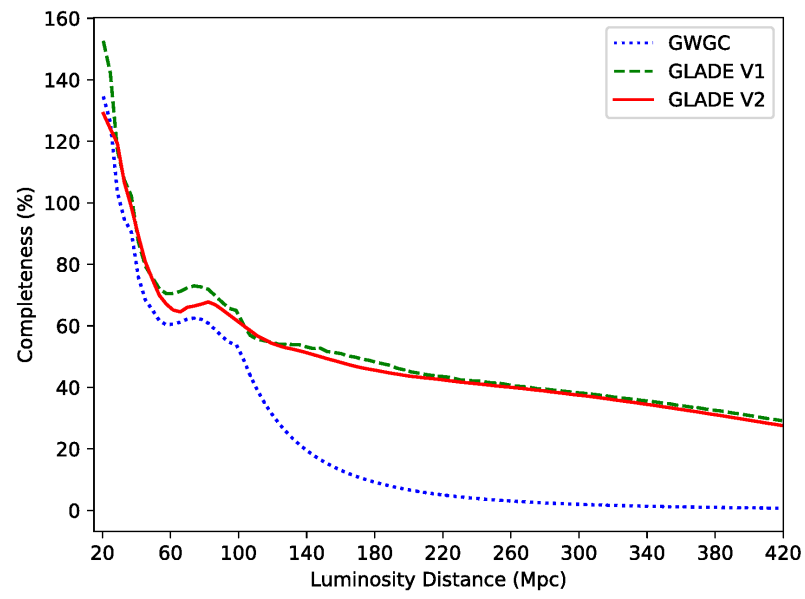

Fig. 2. Comparison of the percentage completeness of GWGC, GLADE V1 and GLADE V2. Completeness is measured as the ratio of cumulative B luminosity in the catalogues to the luminosity expected from a homogeneous complete galaxy catalogue given by Kopparapu et al. (2008). Only galaxies with B magnitude and distance values are included. For GLADE V1, galaxies whose parameters were estimated via regression are excluded.

- When compiling GLADE V1, regression was used to estimate the distance and B magnitude for $>$ 400,000 galaxies. GLADE V2 omits these.

- GLADE V2 adds many new galaxies from the most up to date versions of its constituent catalogues.

- Although many of the newly added galaxies do not have an associated $\mathrm{B}$ magnitude and distance measure, GLADE V2 has comparable completeness to GLADE V1 when galaxies where regression was used to estimate parameters are removed.

\subsection{Galaxy retrieval and ranking}

The first step to retrieving galaxies is to crossmatch a local hdf5 version of the GLADE
V2 galaxy catalogue with the sky map. The ligo.skymap.crossmatch function is used to identify all galaxies within the $99 \%$ localisation region. The Right Ascension, Declination, B magnitude, and distance to these galaxies are stored for ranking. This list is further limited to galaxies within DISTMEAN \pm 5 DISTEST. The galaxies are ranked based on sky map probabilities and the distances and B magnitudes of the galaxies from GLADE V2. The prioritisation algorithm used is based on the approach described by Arcavi et al. (2017). The probability of association of the GW source with a given galaxy is calculated as follows:

1. The location probability measure is given by:

$$
S_{\mathrm{loc}}=p_{\mathrm{loc}} p_{\mathrm{dist}} .
$$

$p_{\text {loc }}$ is the probability that the source is located at the position of the galaxy in the sky map.

The galaxy's distance $D$, taken from the GLADE V2 catalogue, is compared to $\mu_{\text {dist }}$, the estimated distance to the merger within the pixel at the position of the galaxy in the sky map, to calculate $p_{\text {dist }}$ :

$$
p_{\text {dist }}=N_{\text {dist }} \exp \left(\frac{-\left[D-\mu_{\text {dist }}\right]^{2}}{2 \sigma_{\text {dist }}^{2}}\right),
$$

where $N_{\text {dist }}$ is a normalising factor and $\sigma_{\text {dist }}$ is the distance error computed by the BAYESTAR or LALInference algorithm and contained in the pixel at the galaxy's position in the sky map.

2. The brightest and most massive galaxies, where short GRBs are usually found, are ranked higher by using $\mathrm{B}$ band luminosity as a proxy for galaxy mass (Berger 2014). The apparent B magnitude and distance from the filtered GLADE V2 catalogue is used to calculate luminosity, which is 
TABLE 3

OPTIONS AVAILABLE TO THE USER ON THE WEBSITE

\begin{tabular}{|c|c|}
\hline Option & Inputs \\
\hline $\begin{array}{l}\text { Retrieve galaxy list } \\
\text { (Figure } 3 \text { ) }\end{array}$ & $\begin{array}{l}\text { GW source } \\
\text { Percentage localisation re- } \\
\text { gion }(50 \%, 90 \% \text {, or } 99 \%)\end{array}$ \\
\hline $\begin{array}{l}\text { Retrieve galaxy } \\
\text { based on location }\end{array}$ & $\begin{array}{l}+ \\
\text { Longitude } \\
\text { Latitude } \\
\text { Limiting elevation } \\
\text { Time of observation }\end{array}$ \\
\hline $\begin{array}{l}\text { Retrieve galaxy list } \\
\text { based on location } \\
\text { and limiting magni- } \\
\text { tude }\end{array}$ & $\begin{array}{l}+ \\
\text { Limiting magnitude of ob- } \\
\text { servatory }\end{array}$ \\
\hline
\end{tabular}

then folded into the luminosity probability measure $S_{\text {lum }}$ :

$$
S_{\text {lum }}=\frac{L_{\mathrm{B}}}{\sum L_{\mathrm{B}}} .
$$

3. The overall probability of the merger occurring in a galaxy is given by

$$
S=S_{\text {loc }} S_{\text {lum }}
$$

This probability is calculated for all galaxies and then a score is computed by normalising the probabilities to add to 1 .

\section{WEB APPLICATION}

The list of ranked galaxies and their properties is saved to a PostgreSQL database which is hosted on Amazon S3. A web application has been developed to render the outputs of this algorithm publicly post-trigger. To respond to a trigger, PyGCN is used to ingest the GCN alert, begin the galaxy retrieval algorithm and update the website with the new list for the gravitational wave event.

The web application is developed using the Flask web framework which allows for web applications to be written in Python. The site is hosted on Heroku which is equipped with command line access to GitHub, PostgreSQL, and Amazon S3. The 3 options currently available to the user so can be seen in Table 3. For options 2 and 3, instead of inputting their coordinates, the user can choose their observatory from a pre-defined list if it exists in that list. The current options include Boyden Observatory, La Palma, Paranal, and La Silla.

\section{Retrieve galaxy list}

\author{
Select a LIGO/Virgo source and a percentage confidence \\ region to retrieve a list of galaxies within that region:
}

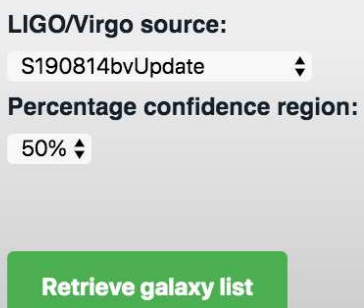

Fig. 3. Screenshot of the 'Retrieve galaxy list' page

The result of these queries is a table of up to 50 galaxies, ranked in order of their probability score. The table contains the galaxy name, probability score $(S)$, Right Ascension, Declination, location probability measure $\left(p_{\text {loc }}\right)$, distance, distance probability measure $\left(p_{\text {dist }}\right)$, B magnitude, B luminosity probability measure $\left(S_{\text {lum }}\right)$ and cumulative probability score. An interactive Aladin DSS image is shown for each galaxy. An example of one entry from the table for S190814bv can be seen in Figure 4. A map of contour regions is also displayed with a plot of all galaxies in the region. The user can download the full table as a json, ascii or txt file.

For option 2, the galaxy list is limited only to galaxies which are visible to the user at their location and time. Visible galaxies are determined using Astroplan (Price-Whelan et al. 2018), and a visibility plot is displayed on the results page. For option 3 , the table contains an extra column, which is an indicator of the detectability of the possible kilonova associated with each galaxy. This is calculated by comparing the minimum detectable source luminosity at the distance of each galaxy with that of a kilonova $\left(M_{\mathrm{KNmin}}=-17\right)$ at the same distance. If the minimum detectable luminosity at that distance is greater than the minimum luminosity of a kilonova, the kilonova is deemed detectable.

\section{DISCUSSION}

\subsection{V0 of the galaxy retrieval algorithm}

V0 of the algorithm was employed during the first half of O3 until November 2019 (O3a), however it was updated for O3b to improve execution time and functionality. A comparison of the features of V0 and V1 of the algorithm can be seen in Table 4. V0 of the algorithm used healpy to read sky 


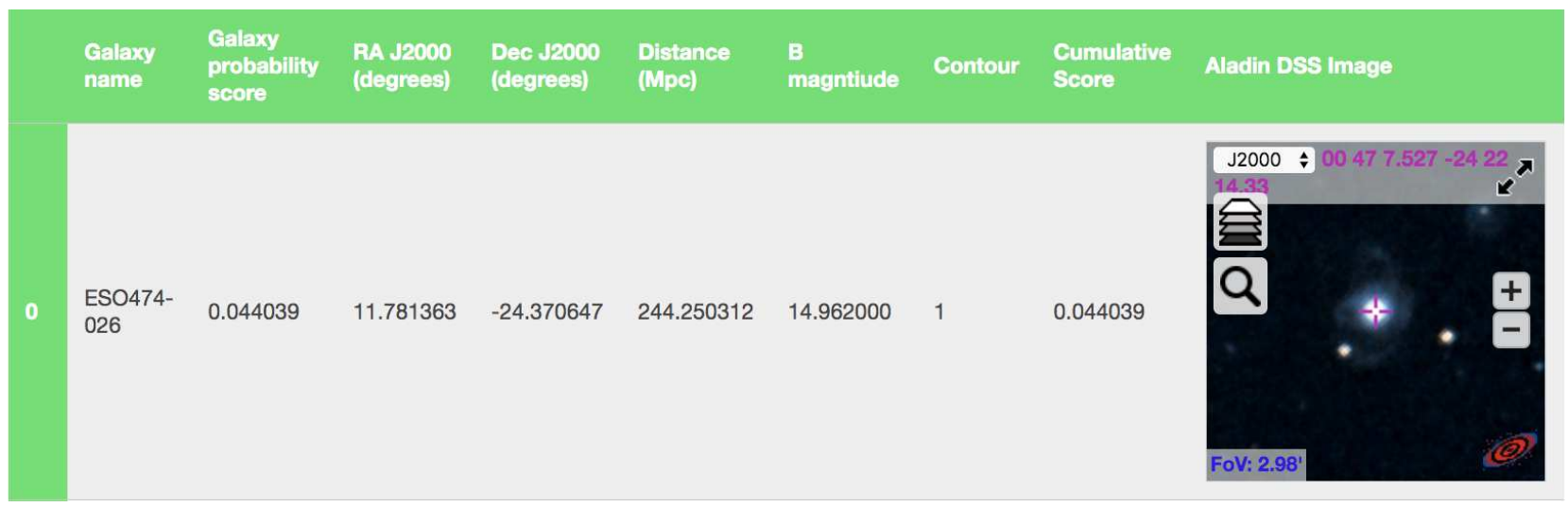

Fig. 4. Screenshot of the table rendered on the results webpage.

TABLE 4

\section{COMPARISON OF V0 AND V1 OF THE GALAXY RETRIEVAL ALGORITHM}

\begin{tabular}{lll}
\hline Property & V0 & V1 \\
\hline Distance limits & \pm DISTSTD & \pm 5 DISTSTD \\
Sky map I/O pack- & healpy & ligo.skymap \\
age used & & \\
$\%$ regions & $50 \%, 90 \%, 99 \%$ & $99 \%$ \\
$\begin{array}{l}\text { Contour identifica- } \\
\text { tion }\end{array}$ & Yes & No \\
\hline
\end{tabular}

maps and the skimage (van der Walt et al. 2014) find_contours method to identify the contours enclosing $99 \%, 90 \%$, and $50 \%$ of the probability within the integrated probability map. The GLADE catalogue was queried in Vizier using Astropy (PriceWhelan et al. 2018) for each contour. MOCPy, a Python package for analysing Multi-Order Coverage (MOC) maps, was used to determine which galaxies were within a contour region. The galaxy lists were refined to galaxies within DISTMEAN \pm DISTSTD. The individual Vizier queries for each contour region, and for the 50\%, $90 \%$ and $99 \%$ regions, lengthened execution time by up to 200 seconds. Therefore, V1 of the algorithm uses ligo.skymap to crossmatch a local hdf5 version of the GLADE catalogue with the sky map in a quick manner. Execution times of V1 of the algorithm for a selection of events from O3 can be seen in Table 5. Additionally, distance limits have increased to \pm 5 DISTSTD to ensure no relevant galaxies are left out.

\subsection{Results in $\mathrm{O} 3$}

Throughout O3 the galaxy retrieval algorithm automatically responded to gravitational wave alerts. The automated system updated the web-
TABLE 5

FEATURES OF SELECTED EVENTS FROM O3

\begin{tabular}{llll}
\hline Event & $\begin{array}{l}99 \% \\
\text { Area }\end{array}$ & \# galaxies & $\begin{array}{l}\text { Execution } \\
\text { time }\end{array}$ \\
\hline S190924h & 1096 & 41,216 & $30 \mathrm{~s}$ \\
S191205ah & 18,565 & 535,928 & $61 \mathrm{~s}$ \\
S191129u & 2211 & 99,445 & $33 \mathrm{~s}$ \\
S190828j & 1278 & 25,651 & $28 \mathrm{~s}$ \\
S190814bv 2137 & 68,266 & $30 \mathrm{~s}$ \\
S190930t & 35,183 & 364,702 & $50 \mathrm{~s}$ \\
S190910d & 7986 & 201,506 & $39 \mathrm{~s}$ \\
\hline
\end{tabular}

site with each new alert or sky map update. The galaxy lists produced by the galaxy retrieval algorithm were used to follow-up the well localised NS-BH event S190814bv. The Telescopio Nazionale Galileo (TNG; D'Avanzo et al. 2019a,b), the WHT (Levan et al. 2019), the Nordic Optical Telescope (NOT; Heintz et al. 2019a,b), the Gamma-Ray Burst Optical/Near-Infrared Detector (GROND; Chen et al. 2019), and the Liverpool Telescope (LT; Perley et al. 2019) followed up 74 galaxies within the $99 \%$ localisation region for this event. These follow-up observations took place within the ElectromagNetic counterparts of GRAvitational wave sources at the VEry Large Telescope (ENGRAVE) collaboration, the GRAvitational Wave Inaf TeAm (GRAWITA; D'Avanzo et al. 2019b,a), the Gravitational Waves at the William Hershel Telescope collaboration (GW@WHT; Levan et al. 2019), and the the the Global Relay of Observatories Watching Transients Happen (GROWTH; Andreoni et al. 2019; Coughlin et al. 2019) collaboration. No counterparts were identified, however these observations 
place constraints on electromagnetic counterparts to NS-BH merger events (Ackley et al. 2020).

\section{CONCLUSIONS}

Our newly developed galaxy retrieval algorithm and web application have responded to all alerts in O3. Each gravitational wave event tests the system, and continuous improvements are being made based on feedback from collaborators and insights from O3. The back-end algorithm is an open source project on GitHub at https://github.com/Lanasalmon/ HOGWARTs, allowing it to be easily integrated into current robotic telescope pipelines. For more details, see Salmon et al. (2020).

Acknowledgements: LS acknowledges the Irish Research Council Postgraduate Scholarship No GOIPG/2017/1525.

We thank the organisers of the 6th workshop on Robotic Autonomous Observatories.

We thank Alberto Castro-Tirado, Morgan Fraser, James Gillanders, Kate Maguire, Owen McBrien, Stephen Smartt and Brian Van Soelen for their useful feedback on the web application.

\section{REFERENCES}

Aasi, J., Abadie, J., Abbott, B. P., et al. 2015, CQGra, 32,5012

Abbott, B. P., Abbott, R., Abbott, T. D., et al. 2018, LRR, 21, 3 2017, ApJ, 848, 12

Acernese, F., Agathos, M., Agatsuma, K., et al. 2015, CQGra, 32, 4001

Ackley, K., Amati, L., Barbieri, C., et al. 2020, A\&A, 643, 113

Alexander, K. D., Margutti, R., Blanchard, P. K., et al. 2018, ApJ, 863, 18

Andreoni, I., Ackley, K., Cooke, J., et al. 2017, PASA, 34,69

Andreoni, I., Goldstein, D. A., Anand, S., et al. 2019, ApJ, 881, 16

Arcavi, I., McCully, C., Hosseinzadeh, G., et al. 2017, ApJ, 848, 33

Arcavi, I., Hosseinzadeh, G., Howell, D. A., et al. 2017, Natur, 551, 64

Barnes, J. \& Kasen, D. 2013, ApJ, 775, 18

Belczynski, K., Perna, R., Bulik, T., et al. 2006, ApJ, 648, 1110

Berger, E. 2014, ARA\&A, 52, 43

Chen, T.-W., Nicuesa Guelbenzu, A., Fraser, M., et al. 2019, GCN, 25372, 1

Connaughton, V., Briggs, M. S., Goldstein, A., et al. 2015, ApJS, 216, 32

Costa, E., Frontera, F., Heise, J., et al. 1997, Natur, 387, 783

Coughlin, M. W., Tao, D., Chan, M. L., et al. 2018, MNRAS, 478, 692
Coughlin, M. W., Ahumada, T., Anand, S., et al. 2019, ApJ, 885, 19

Coulter, D., Foley, R. J., Kilpatrick, C. D., et al. 2017, Sci, 358, 1556

Cowperthwaite, P., Berger, E., Villar, V. A., et al. 2017, ApJ, 848, 17

Dálya, G., Galgóczi, G., Dobos, L. 2018, MNRAS, 479, 2374

D'Avanzo, P., Rossi, A., Greco, G., et al. 2019a, GCN, 25361, 1

D'Avanzo, P., Melandri, A., Izzo, L., et al. 2019b, GCN, 25331, 1

Dobie, D., Murphy, T., Kaplan, D., et al. 2019, PASA, 36, 19

Drout, M., Piro, A. L., Shappee, B. J., et al. 2017, Sci, 358,1570

Eichler, D., Livio, M., Piran, T., \& Schramm, D. N. 1989, Natur, 340, 126

Evans, P. A., Kennea, J. A., Palmer, D. M., et al. 2016, MNRAS, 462, 1591

Evans, P. A., Cenko, S. B., Kennea, J. A., et al. 2017, Sci, 358, 1565

Fong, W., Margutti, R., Chornock, R., et al. 2016, ApJ, 833,151

Fong, W., Blanchard, P. K., Alexander, K. D. et al. 2019, ApJ, 883, 1

Frail, D., Kulkarni, S. R., Nicastro, L., Feroci, M., \& Taylor, G. 1997, Natur, 389, 261

Gehrels, N., Cannizzo, J. K., Kanner, J., et al. 2016, ApJ, 820, 136

Goad, M., Tyler, L. G., Beardmore, A. P., et al. 2007, A\&A, 476, 1401

Goldstein, A., Veres, P., Burns, E., et al. 2017, ApJ, 848, 14

Górski, K. M., Hivon, E., Banday, A. J., et al. 2005, ApJ, 622, 759

Granot, J., Panaitescu, A., Kumar, P., \& Woosley, S. E. 2002, ApJ, 570, 61

Heintz, K. E., Malesani, D. B., Leloudas, G., et al. 2019a, GCN, 25376, 1

Heintz, K. E., Malesani, D. B., Selsing, J., et al. 2019b, GCN, 25325, 1

Hotokezaka, K. \& Piran, T. 2015, MNRAS, 450, 1430

Jin, Z.-P., Li, X., Wang, H., et al. 2018, ApJ, 857, 128

Kasen, D., Badnell, N. R., \& Barnes, J. 2013, ApJ, 774, 25

Kasliwal, M. M., Nakar, E., Singer, L. P., et al. 2017, Sci, 358, 1559

Kathirgamaraju, A., Barniol Duran, R., \& Giannios, D. 2018, MNRAS, 473, 121

Kilpatrick, C. D., Foley, R. J., Kasen, D., et al. 2017, Sci, 358,1583

Klingler, N. J., Kennea, J. A., Evans, P. A., et al. 2019, ApJS, 245, 15

Kopparapu, R. K., Hanna, C., Kalogera, V., et al. 2008, ApJ, 675, 1459

Lazzati, D., Perna, R., Morsony, B. J., et al. 2018, PhRvL, 120, 1103 
Levan, A. J., Fraser, M., Jonker, P., et al. 2019, GCN, 25332, 1

Li, L.-X. \& Paczyński, B. 1998, ApJ, 507, 59

LIGO Scientific Collaboration. 2018, LIGO Algorithm Library - LALSuite, free software (GPL)

Makarov, D., Prugniel, P., Terekhova, N., Courtois, H., \& Vauglin, I. 2014, A\&A, 570, 13

Margutti, R., Berger, E., Fong, W., et al. 2017, ApJ, 848, 20

McCully, C., Hiramatsu, D., Howell, D. A., et al. 2017, ApJ, 848, 32

Mereghetti, S. 2013, An INTEGRAL view of the highenergy sky (the first 10 years)-9th INTEGRAL Workshop and celebration of the 10th anniversary of the launch, Vol. 176, SISSA Medialab, 126

Metzger, B. D. 2017, LRR, 20, 3

Metzger, B. D. \& Berger, E. 2012, ApJ, 746, 48

Mooley, K. P., Nakar, E., Hotokezaka, K., et al. 2018, Natur, 554, 207

Narayan, R., Paczynski, B., \& Piran, T. 1992, ApJ, 395, 83

Perley, D. A., Copperwheat, C. M., Malesani, D. B., Levan, A., \& Growth Collaboration. 2019, GCN, 25328, 1

Pian, E., D'Avanzo, P., Benetti, S., et al. 2017, Natur, 551,67

Pozanenko, A., Chernenko, A., Beskin, G., et al. 2003, ASPC, 295, 457

Price-Whelan, A. M., Hogg, D. W., Rix, H.-W., et al. 2018, AJ, 156, 18

Rana, J. \& Mooley, K. P. 2019, arXiv preprint arXiv:1904.07335
Rana, J., Singhal, A., Gadre, B., Bhalerao, V., \& Bose, S. 2017, ApJ, 838, 108

Roberts, L. F., Kasen, D., Lee, W. H., \& Ramirez-Ruiz, E. 2011, ApJ, 736, 21

Salmon, L., Hanlon, L., Jeffrey, R. M., \& Martin-Carrillo, A. 2020, A\&A, 634, 32

Savchenko, V., Ferrigno, C., Kuulkers, E., et al. 2017, ApJ, 848, 15

Singer, L. P. \& Price, L. R. 2016, PhRvD, 93, 4013

Skrutskie, M. F., Cutri, R. M., Stiening, R., et al. 2006, AJ, 131, 1163

Smartt, S. J., Chen, T.-W., Jerkstrand, A., et al. 2017, Natur, 551, 75

Tanaka, M. \& Hotokezaka, K. 2013, ApJ, 775, 113

Tanvir, N. R., Levan, A. J., González-Fernández, C., et al. 2017, ApJ, 848, 27

Troja, E., Piro, L., Ryan, G., et al. 2018, MNRAS, 478, 18

Valenti, S., Sand, D. J., Yang, S., et al. 2017, ApJ, 848, 24

van der Walt, S., Schönberger, J. L., Nunez-Iglesias, J., et al. 2014, arXiv e-prints, arXiv:1407.6245

Van Paradijs, J., Groot, P. J., Galama, T., et al. 1997, Natur, 386, 686

Veitch, J., Raymond, V., Farr, B., et al. 2015, PhRvD, 91, 2003

White, D. J., Daw, E. J., \& Dhillon, V. S. 2011, CQGra, 28,5016

Wu, Y. \& MacFadyen, A. 2018, ApJ, 869, 55

Yang, S., Sand, D. J., Valenti, S., et al. 2019, ApJ, 875, 59 